\title{
Application of Standard Models and Artificial Neural Network for Missing Rainfall Estimation
}

\author{
Madhuri Dubey ${ }^{1 *}$ and M.K. Hardaha ${ }^{2}$ \\ ${ }^{1}$ Indian Institute of Technology, Kharagpur, West Bengal, India \\ ${ }^{2}$ College of Agricultural Engineering, J.N.K.V.V., Jabalpur, Madhya Pradesh, India \\ *Corresponding author
}

\begin{tabular}{|l|}
\hline K e y w o r d s \\
Arithmetic mean \\
model, Normal ratio \\
model, Inverse \\
distance model, \\
Artificial neural \\
network
\end{tabular}

\section{Introduction}

Precipitation plays a significant role in agriculture and it is the most important part of climatological studies (Ayoade, 1983). The study about precipitation is important due to various reasons, such as identifying
Precipitation records often suffer from missing data values for certain time periods due to various reasons, one of them being the malfunctioning of rain gauges. This is an important issue in practical hydrology as it affects the continuity of rainfall data. The missing data values ultimately influence the results of hydrologic studies that use rainfall data as one of the input variables. Therefore, it is crucial to estimate the missing rainfall data for qualitative hydrologic assessment. In this study, the annual rainfall data of eight districts of the state Madhya Pradesh, India is collected in the time frame of the year 1901 to 2011. The collected information is used for estimating missing annual rainfall data. Various existing standard models, such as arithmetic mean, normal ratio, inverse distance weighting, multiple linear regression as well as unconventional methods like artificial neural network (ANN) is used and compared to determine missing rainfall records in the collected data. The results as obtained show that among the various standard models, multiple linear regression models perform better. The model is validated and the correlation coefficient (R), root mean square error (RMSE) and mean absolute error (MAE) are found to be $0.913,9017 \mathrm{~mm}$, and $49.7 \mathrm{~mm}$, respectively. When the ANN model is applied for estimating annual missing rainfall data, it is found that Levenverg Marquardt $(\mathrm{lm})$ algorithm with 7 neurons and 50-year length of records performs better than the other combination of algorithms, neurons and length of records. During the training of this model, the values of R, RMSE and MAE value are found to be 0.998 , $4.4 \times 10^{-4} \mathrm{~mm}$ and $53.047 \mathrm{~mm}$, respectively, and during validation, they are $0.858,1.667$ $\mathrm{mm}$ and $49.103 \mathrm{~mm}$, respectively. The results as obtained indicate that the ANN method is most suitable for estimating the missing annual rainfall data. 
For the effective study and analysis of precipitation, the consistency and continuity of the rainfall data are very crucial. Both consistency and continuity may be disturbed due to change in observational procedure and incomplete records (missing observations), which may vary in length ranging from one or two days to decades of years. The rainfall data are mainly time series data which are essential for the hydrological design of various structures, such as dams and bridges. Any disruption in the rainfall data may result in the failure of these structures resulting in major social and economic loss.

For filling up such disrupted time series data, existing literature consists of various standard and advanced techniques, such as arithmetic method, inverse distance weighting, normal ratio method, multiple linear regression, spatial interpolation methods, integrating surface interpolation techniques and spatiotemporal association rules based methods techniques (Teegavarapu, 2009; Kim and Pachepsky, 2010; Nkuna and Odiyo, 2011; Kajornrit et al., 2011; Piazza et al., 2011; Chen and Liu, 2012).

Kim and Pachepsky (2010) used regression tree with artificial neural network for infilling daily precipitation data for Soil and Water Assessment Tool (SWAT) streamflow simulation. Four methods local mean, normal ratio, inverse distance, and aerial ratio precipitation method were compared by Silva et al., (2007) for estimating monthly missing rainfall for the different agro-ecological zone of the Sri Lanka, and they found that different methods are suitable for different regions.

Piazza et al., (2011) compared different techniques, such as inverse distance weighting, simple linear regression, multiple regressions, geographically weighted regression, artificial neural networks, and geostatistical models, such as ordinary kriging and residual ordinary kriging for spatial interpolation of rainfall data to create a serially complete monthly time series of precipitation for Sicily, Italy. The results reveal that residuals ordinary kriging perform best at monthly and annual scale to complete monthly time series. Artificial neural network method is also successfully used by researchers in many scientific and engineering disciplines since they are capable of correlating large and complex multiparameter dataset without any prior knowledge of the relationship between the parameters. Applications of different types of artificial neural network were shown by many researchers for estimation of missing rainfall data (Bustami et al., 2007; Nkuna and Odiyo 2011; Nourani et al., 2012; Terzi and Cevik 2012).

The suitability of different methods may vary from one region to another as it is utilized by many investigators in different places of the world (references). Hence, the aim of this study is to estimate missing annual rainfall data using different models such as arithmetic mean model (AMM), normal ratio model (NRM), inverse distance model (IDM), multiple linear regression model (MLR) and artificial neural network (ANN).

In this study, the annual rainfall data of eight districts of the state Madhya Pradesh, India is collected in the time frame of the year 1901 to 2011. The eight districts are Mandla, Seoni, Narsinghpur, Damoh, Umaria, Dindori, Katni, Jabalpur. The complete rainfall data is used to estimate the missing rainfall of one of the district, viz. Jabalpur district. The remaining seven districts were selected because of their similar climatology with the Jabalpur district. This study will produce reliable missing rainfall data that may be ultimately used in hydrological modeling and water resources planning and management. 


\section{Materials and Methods}

\section{Study area and data used}

For the study, eight districts of the Madhya Pradesh situated at the central part of the India, is selected, as shown in Figure 1. The Madhya Pradesh has a subtropical climate with extreme summer and winter seasons indicating high variability, as well as high variability in rainfall with either extreme rain or drought. The average annual rainfall of this state is around $1370 \mathrm{~mm}$. The south-eastern districts of the state mostly receive heavy rainfall. The state receives maximum rainfall as $2150 \mathrm{~mm}$ and minimum as $1000 \mathrm{~mm}$, and its magnitude decreases from east to west. The annual rainfalls of all the state districts range from $1038 \mathrm{~mm}$ to $1245 \mathrm{~mm}$.

The rainfall data of the selected eight districts of Madhya Pradesh, viz. Jabalpur, Katni, Narsinghpur, Seoni, Mandla, Damoh, Umaria, and Dindori for the period of 110 years (January 1901 to December 2011, excluding 2003) have been collected from the secondary data sources, such as India Water Portal and India Meteorology Department, Pune. The rainfall data of Jabalpur district is assumed to be missing and is to be estimated by using the rainfall data of the surrounding districts. Standard models and ANN is developed for the estimation of annual missing rainfall data of Jabalpur district. Out of 110 years length record of the rainfall, 70 years of data is used for calibration and 40 years data is used for validation of the developed model.

\section{Models for estimating missing rainfall data}

In the present study, the standard models and ANN have been used for estimating missing annual rainfall of Jabalpur district based on the rainfall data of surrounding seven districts, viz. Mandla, Katni, Seoni, Narsinghpur, Damoh, Dindori, and Umaria.
The various models applied for the study are the arithmetic mean, normal ratio, inverse distance, multiple linear regression, and ANN, and are briefly explained below:

\section{Arithmetic mean model}

Arithmetic mean model is used to estimate the missing observation of station $\mathrm{X}$, if normal annual precipitations at surrounding gauges vary within the range of $10 \%$ of the normal annual precipitation (Chow et al., 1988). This model is given by Eq. (1):

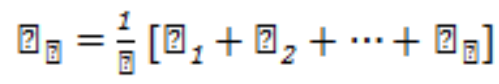

\section{Normal ratio model}

Normal ratio model is used if any surrounding gauges have the normal annual precipitation exceeding $10 \%$ of the considered gauge. The missing data are estimated by Eq. (2).

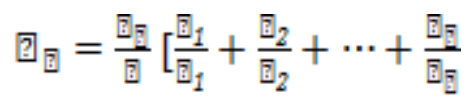

\section{Inverse distance model}

In this model, the weight for each sample is inversely proportionate to its distance from the point being estimated and is given in Eq. (3).

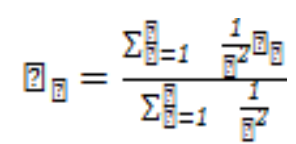

Where $\mathrm{R} \mathrm{x}$ is rainfall missing data at station $\mathrm{X}$, and $\mathrm{R} 1, \mathrm{R} 2$, and $\mathrm{Rn}$ are rainfall at the station 1, 2 and n, respectively. Nx, N1, N2, and $\mathrm{Nn}$ are normal annual precipitation at the station $\mathrm{X}, 1,2$ and $\mathrm{n}$, respectively. $\mathrm{M}$ is a number of stations and $\mathrm{d}$ is the distance between station (where data is missing) and surrounding stations. 


\section{Multiple linear regression model}

Regression analysis is used for explaining or modeling the relationship between a single variable $\mathrm{y}$, called the response, output or dependent variable, and one or more predictor, input, independent or explanatory variables, $\mathrm{x} 1 \ldots \ldots \ldots . . . \mathrm{xn}$. When the number of predictor variables, $\mathrm{n}=1$, it is called simple regression, but when $\mathrm{n}>1$, it is called multiple regression or sometimes multivariate regression. Assume that two precipitation gauges $\mathrm{y}$ and $\mathrm{x}$ have long records of annual precipitation, i.e. $\mathrm{y} 1, \mathrm{y} 2, \ldots \mathrm{y} n$ and $\mathrm{x} 1, \mathrm{x} 2, \ldots$ $\mathrm{x} \mathrm{n}$. The precipitation yt is missing and $\mathrm{x}$ and $\mathrm{y}$ are sample means. The missing data can be filled in based on a simple linear regression model. The model can be written as in Eq (4):

圆=国国国十回

Where $\mathrm{a}$ and $\mathrm{b}$ are regression coefficients.

\section{Artificial Neural Network (ANN)}

The feed-forward neural network is selected for the analysis, wherein the input data (rainfall at surrounding stations) are fed into the nodes which pass the hidden nodes after getting multiplied by the weight. The hidden layer neurons are selected using trial and error procedure. The output neurons of the ANN provide the missing value at the stations other than the station of interest. The rainfall data of the selected Jabalpur district station for the period of 1901-1971 and 1971-2011 is used for training and validation purpose. For developing ANN model for estimation of missing annual rainfall, initially, the model is trained with 12 training algorithm, 5 neurons and 30-year length of the record. The training algorithm, which performed best, has been considered for further refinement of the model with a varying number of neurons and varying length of data set. Neurons in the hidden layer have been varied from 1 to 10 , and the number of neurons which performed best is considered for further improvement of the ANN model. Thereafter, the model is trained by the various length of a years (30, 50 and 70) for checking the sensitivity of the ANN model to the size of training data. Mean and standard deviation (mapstd) function is used for scaling all input and target data. Initially, 5 numbers of neurons are included in the single hidden layer of the model. The objective of training the model is to achieve the minimum mean error between estimated and targeted rainfall. The neural network utility file is edited in highly efficient computer programming software, MATLAB Version 6.5. The input data source file, network option, training function, setting for the data for training, validation, plotting the predicting values and saving the network is created and run in the software.

\section{Model comparison}

The performance of all the applied models is evaluated by three effective performance measures, viz. Root Mean Squared Error (RMSE), Mean Absolute Error (MAE) and Correlation coefficient (R). R measures the degree to which two variables are linearly related. RMSE and MAE provided a balanced perspective of the goodness of fit as moderate output values (Karunanithi et al., 1994).

\section{Results and Discussion}

\section{Performance of standard models}

The derived mathematical forms of normal ratio model, inverse distance model, and multiple linear regression models for determining the missing rainfall, are given by Eq. (5), (6) and (7), respectively.

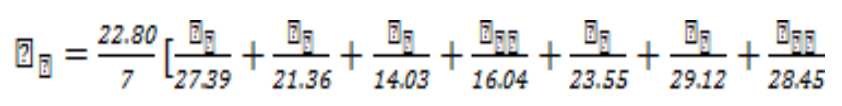




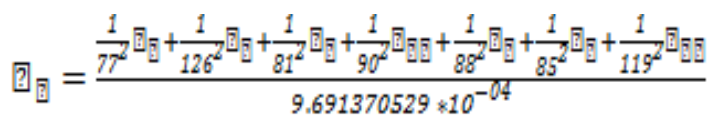

$\square_{\mathrm{B}}=\left(0.8521109 * \mathrm{Q}_{\mathrm{g}}\right)+\left(0.075227 * \mathrm{Q}_{\mathrm{g}}\right)+\left(0.1128 * \mathrm{Q}_{\mathrm{B}}\right)+\left(0.060483 * \mathrm{Q}_{\mathrm{B}}\right)$ $+\left(0.083371 * \square_{\mathrm{q}}\right)+\left(0.529176 * \mathrm{\Xi}_{\mathrm{q}}\right)+\left(-0.76742 * \mathrm{\Xi}_{\mathrm{B}}\right)+(-6.82664)$

Where, $\mathrm{P}_{\mathrm{X}}, \mathrm{P}_{\mathrm{M}}, \mathrm{P}_{\mathrm{S}}, \mathrm{P}_{\mathrm{N}}, \mathrm{P}_{\mathrm{DA}}, \mathrm{P}_{\mathrm{K}}, \mathrm{P}_{\mathrm{U}}$ and $\mathrm{P}_{\mathrm{DI}}$ are the annual rainfall $(\mathrm{mm})$ at the eight districts, viz. Jabalpur, Mandla, Seoni, Narsinghpur, Damoh, Katni, Umaria, and Dinori, respectively.

Performance of these models for training and validation is summarized in Table 1 . The results showed that MLR performed better over the AMM, NRM, and IDW, as it has a lower value of RMSE and MAE for both training and validation. Figure 2 represents the scatter plot of observed and estimated rainfall for the applied standard models. It is observed from the Figure 2 that the estimated rainfall is more closely related to observed rainfall in case of MLR model as compared to the other models.

The performance of the standard models were in following order MLR $>$ IDM $>$ AMM $>N R M$. These results are supported by Sattari et al., (2017) in which multiple linear regressions were proved to be the best among the inverse distance, normal ratio, single estimator and non-linear iterative partial least squares algorithm models. However, inverse distance, normal ratio, and arithmetic mean method is also found to be efficient to capture missing rainfall data in Sri Lanka (Silva et al., 2007).

\section{Performance of artificial neural network}

Performance of the ANN model, developed with different training algorithms during model training and validation, are shown in Table 2. Model with Levenverg-Marquardt algorithm performed best as it has a lower value of RMSE and MAE than other models trained by a different algorithm. By critical evaluation of the performance indicators, it can be stated that the ANN model trained with "trainlm" training algorithm performed better than the other models trained by other algorithms. The Model with "trainlm" algorithm was further optimized for calculating the optimal number of neurons in the hidden layer.

The ANN model with learning function "trainlm" of normalization function "mapstd" with 30 years data set has been trained with 1 to 10 numbers of neurons and evaluated for an optimum number of neurons. Performance of ANN model developed with a different number of neurons during model training and its validation are shown in Table 3 . From the Table 3, it is found that the performance of the ANN model is the best with 7 neurons and it is further refined for a different length of data set.

The performance of the ANN model developed with various lengths of data is shown in Table 4. From the Table 4, it is observed that the model with $\mathrm{L}=70$ performed better than other ANN models. ANN model with "trainlm" learning function, 7 neurons and trained with 50-year data set for estimation of missing rainfall of annual rainfall of Jabalpur district is better than other combination of algorithm, a number of neurons and length of records. It has the lowest RMSE and MAE as $4.109 \mathrm{~mm}$ and $3.286 \mathrm{~mm}$, respectively during training and $86.254 \mathrm{~mm}$ and $49.103 \mathrm{~mm}$, respectively during the validation of the model. Furthermore, it showed good R-value during training and validation which is 0.999 and 0.913 , respectively.

Figure 3 represents the comparison of estimated and observed rainfall with the selected combination of ANN model for 50 years of training and 40 years of validation period. 
Table.1 Performance of Standard models for annual rainfall for validation

\begin{tabular}{|c|c|c|c|c|c|c|c|c|}
\hline & \multicolumn{9}{|c|}{ Training } & \multicolumn{4}{c|}{ Validation } \\
\hline Indicators & AMM & IDM & NRM & MLR & AMM & IDM & NRM & MLR \\
\hline R & 0.92 & 0.94 & 0.95 & 0.97 & 0.90 & 0.91 & 0.90 & 0.91 \\
\hline $\begin{array}{c}\text { RMSE } \\
(\mathrm{mm})\end{array}$ & 85.40 & 80.40 & 185.50 & 80.40 & 101.90 & 97.60 & 213.30 & 94.00 \\
\hline MAE $(\mathrm{mm})$ & 52.60 & 47.60 & 175.20 & 40.40 & 62.50 & 58.50 & 187.50 & 49.70 \\
\hline
\end{tabular}

Table.2 Performance of ANN model with various training algorithm for annual rainfall

\begin{tabular}{|c|c|c|c|c|c|c|}
\hline \multirow{2}{*}{ Algorithm } & \multicolumn{2}{|c|}{$\mathrm{R}$} & \multicolumn{2}{c|}{$\begin{array}{c}\text { RMSE } \\
(\mathrm{mm})\end{array}$} & \multicolumn{2}{c|}{$\begin{array}{c}\text { MAE } \\
(\mathrm{mm})\end{array}$} \\
\cline { 2 - 7 } & trg & val & trg & val & trg & val \\
\hline trainlm & 0.99 & 0.917 & 6.55 & 93.69 & 5.18 & 52.31 \\
\hline traingd & 0.98 & 0.886 & 38.65 & 113.35 & 31.94 & 76.79 \\
\hline traingdm & 0.96 & 0.872 & 62.97 & 115.46 & 48.81 & 83.73 \\
\hline traingda & 0.96 & 0.797 & 82.08 & 140.25 & 68.79 & 103.27 \\
\hline traingdx & 0.98 & 0.858 & 39.54 & 127.71 & 31,94 & 79.50 \\
\hline traincgf & 0.94 & 0.872 & 82.51 & 118.95 & 65.99 & 76.68 \\
\hline traincgp & 0.92 & 0.825 & 105.16 & 137.25 & 85.48 & 110.40 \\
\hline traincgb & 0.98 & 0.912 & 47.04 & 99.68 & 36.49 & 66.36 \\
\hline trainscg & 0.98 & 0.875 & 52.95 & 114.64 & 35.72 & 76.24 \\
\hline trainbfg & 0.99 & 0.895 & 33.36 & 105.49 & 26.34 & 63.03 \\
\hline trainoss & 0.98 & 0.882 & 40.05 & 113.27 & 29.55 & 68.68 \\
\hline trainrp & 0.96 & 0.861 & 69.16 & 119.15 & 53.36 & 87.73 \\
\hline
\end{tabular}

Table.3 Performance of ANN model with different number of neurons for annual rainfall

\begin{tabular}{|c|c|c|c|c|c|c|c|}
\hline \multirow[t]{2}{*}{ S.N. } & \multirow{2}{*}{$\begin{array}{l}\text { Number } \\
\text { of } \\
\text { Neurons }\end{array}$} & \multicolumn{2}{|c|}{$\mathrm{R}$} & \multicolumn{2}{|c|}{$\begin{array}{l}\text { RMSE } \\
(\mathrm{mm})\end{array}$} & \multicolumn{2}{|c|}{$\begin{array}{l}\text { MAE } \\
(\mathrm{mm})\end{array}$} \\
\hline & & $\operatorname{trg}$ & val & $\operatorname{trg}$ & $\mathrm{val}$ & $\operatorname{trg}$ & val \\
\hline 1. & N1 & 0.974 & 0.830 & 96.63 & 121.89 & 66.63 & 99.82 \\
\hline 2. & $\mathrm{~N} 2$ & 0.979 & 0.830 & 94.10 & 132.40 & 75.60 & 106.00 \\
\hline 3. & N3 & 0.990 & 0.923 & 19.16 & 91.20 & 15.20 & 58.44 \\
\hline 4. & $\mathrm{~N} 4$ & 0.983 & 0.902 & 6.89 & 102.50 & 4.34 & 59.13 \\
\hline 5. & N5 & 0.991 & 0.917 & 6.55 & 93.60 & 5.18 & 51.98 \\
\hline 6. & N6 & 0.989 & 0.910 & 9.99 & 92.41 & 8.25 & 54.38 \\
\hline 7. & N7 & 0.997 & 0.807 & 5.20 & 89.01 & 3.62 & 51.63 \\
\hline 9. & N9 & 0.993 & 0.919 & 9.02 & 98.84 & 6.08 & 58.37 \\
\hline 10. & N10 & 0.992 & 0.889 & 48.40 & 114.70 & 41.40 & 79.47 \\
\hline
\end{tabular}


Table.4 Performance of ANN model with varying length of record for annual rainfall

\begin{tabular}{|c|c|c|c|c|c|c|c|}
\hline S.N. & Length of & \multicolumn{2}{|c|}{$\mathrm{R}$} & \multicolumn{2}{c|}{ RMSE $(\mathrm{mm})$} & \multicolumn{2}{c|}{ MAE $(\mathrm{mm})$} \\
\cline { 3 - 8 } & Records (L) & Trg & val & trg & val & trg & val \\
\hline 1. & L-30 & 0.997 & 0.807 & 5.20 & 89.01 & 3.62 & 51.63 \\
\hline 2. & L-50 & 0.999 & 0.913 & 4.10 & 86.25 & 3.28 & 49.10 \\
\hline 3. & L-70 & 0.998 & 0.858 & 7.85 & 104.26 & 6.12 & 53.04 \\
\hline
\end{tabular}

Fig.1 Index map of Madhya Pradesh showing selected districts

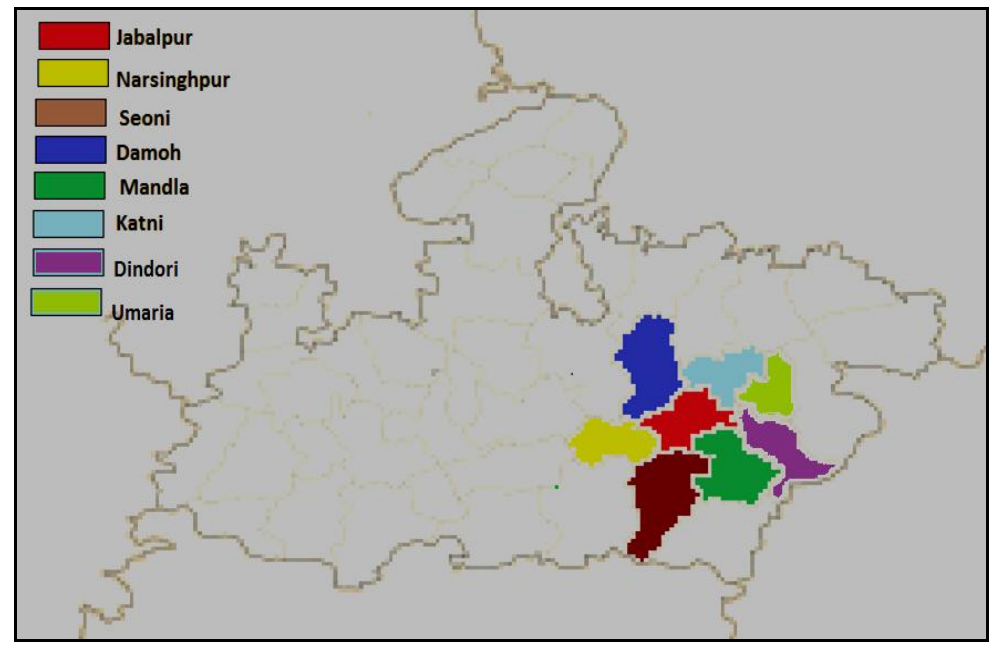

Fig.2 Relationship between observed and estimated rainfall given by arithmetic mean model and normal ratio model inverse distance model and multiple linear regression models for Annual rainfall
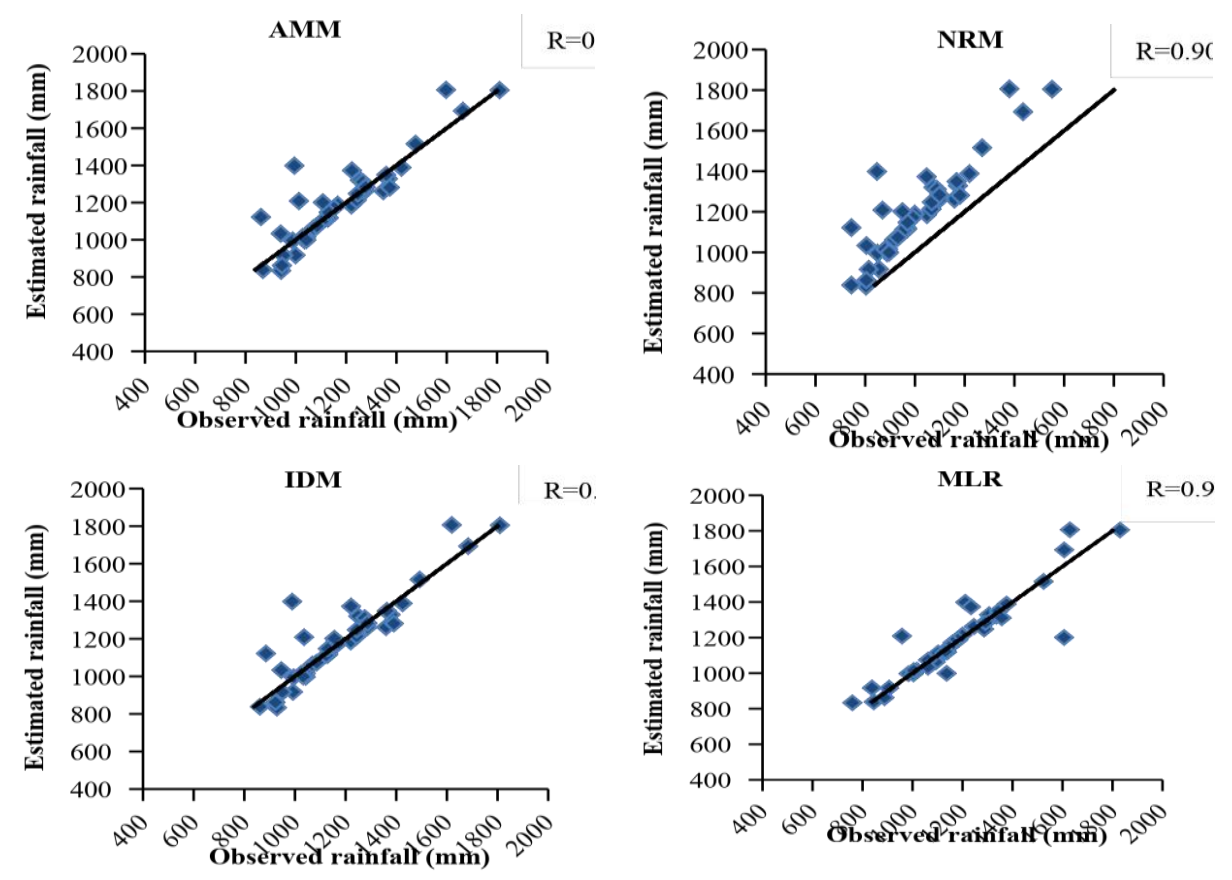
Fig.3 Rainfall graph showing estimated and observed rainfall, $N=7$, (training with 50 year data) for annual rainfall

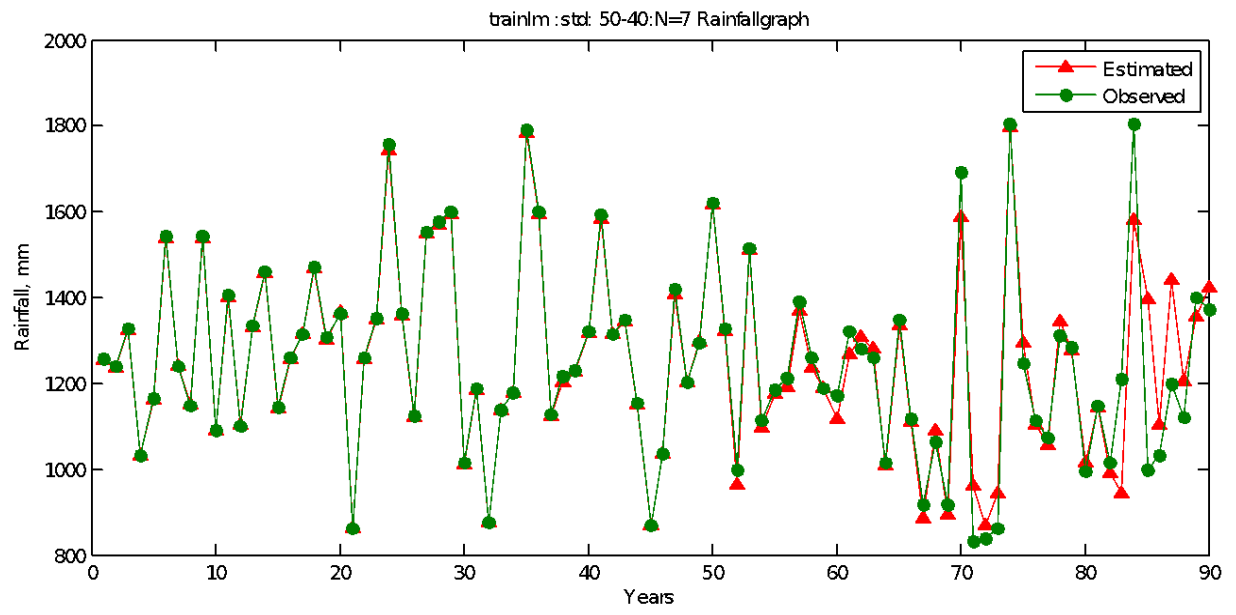

It is depicted from Figure 3 that in most of the years, the estimated value of rainfall matches with the observed value. The similar outcome was found in the study conducted by Ghuge and Regulwar (2013) in Maharashtra, India where ANN was effectively used for estimating missing rainfall in Maharashtra.

\section{Comparison of standard and ANN models}

From the used standard model MLR performed slightly well compared to other standard models. Further comparison with the developed ANN model showed that ANN is more effective than the standard models. From Tables 1 and 4, it can be concluded that ANN has a higher capability of prediction. By comparing the statistics obtained from standard models with the best ANN combination, it can be concluded that ANN has the lowest RMSE, MAE and higher R-value for estimating missing rainfall at the Jabalpur district.

In conclusion, rainfall continuity and consistency are the two keys of viable hydrological analysis and design of the hydrological structure. Maintaining their continuity is important by estimating the missing rainfall data. Therefore, in this study, the missing annual rainfall of Jabalpur district is estimated using four standard models, viz. AM,
NRM, IDW and MLR, and advance model, ANN. ANN models were optimized in respect of learning algorithm, number of neurons and length of data set used for training. These models have been compared based on the various performance indicators. Both standard models and ANN model have the ability to estimate the missing rainfall data. However, in the case of the standard model, MLR performed best over the other standard models with lowest RMSE and MAE values and with highest Rvalue. In the case of ANN, the model developed with the Levenverg Marquardt algorithm, 50year length of record performed well with 5 neurons as it showed the lowest error with higher R-value. In addition, it is evident from performance indicators that the standard models show greater errors as compared to the ANN model. Hence, it may be concluded that the ANN model is a most effective method for estimating the missing annual rainfall data. This study can be further extended for the estimation of monthly and daily missing rainfall data.

\section{References}

Ayoade, J.O. 1983. Introduction to Climatology for the Tropics. John Wiley and Sons: New York.

Bustami, R., Bessaih, N., Bong, C., and Suhaili, S. 2007. Artificial Neural Network for 
Precipitation and Water Level Predictions of Bedup River. IAENG International Journal of computer science, 34(2).

Chen, F-W, and Liu C-W 2012. Estimation of the spatial rainfall distribution using inverse distance weighting (IDW) in the middle of Taiwan. Paddy and Water Environment, 10(3), 209-222. doi:10.1007/s10333-012-0319-1.

Chow, V.T., Maidment, D.R. and Mays, L.W. 1988. Applied hydrology, McGraw Hill Book Company, ISBN 0-07-010810-2.

Ghuge, H.K., and Regulwar, D.G. 2013. Artificial neural network method for estimation of missing data. International Journal of Advanced Technology in Civil Engineering, 2, 1-4.

Kajornrit, J., Wong, K.W., and Fung, C.C. 2011. Estimation of missing rainfall data in northeast region of Thailand using spatial interpolation methods. Australian Journal of Intelligent Information Processing Systems, 13(1).

Karunanithi, N.G., Whitley, D. and Bovee, K.1994. Neural network for river flow prediction. ASCE J. Comp. Civil Engg. 8(2), 201-220.

Kim, J.W., and Pachepsky, Y.A. 2010. Reconstructing missing daily precipitation data using regression trees and artificial neural networks for SWAT streamflow simulation. Journal of hydrology, 394(3-4), 305-314.

Nkuna, T.R., and Odiyo, J.O. 2011. Filling of missing rainfall data in Luvuvhu River Catchment using artificial neural networks. Physics and Chemistry of the Earth, Parts A/B/C, 36(14-15), 830-835. doi:10.1016/j.pce.2011.07.041.

Nourani, V. 2012. Investigating the Ability of Artificial Neural Network (ANN) Models to Estimate Missing Rain-gauge Data. Journal of Environmental Informatics, 19(1), 38-50. doi:10.3808/jei.201200207.

Piazza, A., Conti, F.L., Noto, L.V., Viola, F., and La Loggia, G. 2011. Comparative analysis of different techniques for spatial interpolation of rainfall data to create a serially complete monthly time series of precipitation for Sicily, Italy. International Journal of Applied Earth Observation and Geoinformation, 13(3), 396-408.doi:10.1016/j.jag.2011.01.005.

Sattari, M.T., Rezazadeh-Joudi, A., and Kusiak, A. 2017. Assessment of different methods for estimation of missing data in precipitation studies. Hydrology Research, 48(4), 1032-1044. https://doi.org/10.2166/nh.2016.364.

Silva, R.P., Dayawansa, N.D.K, and Ratnasiri, M.D. 2007. A comparison of methods used in estimating missing rainfall data. Journal of Agricultural Sciences, 3(2), 101. doi:10.4038/jas.v3i2.8107.

Teegavarapu, R.S.V. 2009. Estimation of missing precipitation records integrating surface interpolation techniques and spatio-temporal association rules. Journal of Hydroinformatics, 11(2), 133-146. doi:10.2166/hydro.2009.009.

Zhang, M., Fulcher, J., and Scofield, R.A. 1997. Rainfall estimation using artificial neural network group. Neurocomputing, 16(2), 97-115. doi:10.1016/s09252312(96)00022-7.

\section{How to cite this article:}

Madhuri Dubey and Hardaha, M.K. 2019. Application of Standard Models and Artificial Neural Network for Missing Rainfall Estimation. Int.J.Curr.Microbiol.App.Sci. 8(01): 1564-1572. doi: https://doi.org/10.20546/ijcmas.2019.801.164 